\title{
Development of a method for quantitative determination and preliminary pharmacokinetics study of a new anticancer agent
}

A. A. Okhina ${ }^{1,2}$, A. D. Rogachev ${ }^{1,2}$, K. S. Kovaleva ${ }^{1,2}$, O. I. Yarovaya ${ }^{1,2}$, A. S. Khotskina ${ }^{3}$, E. L. Zavyalov ${ }^{3}$, A. G. Pokrovsky ${ }^{2}$, N. F. Salakhutdinov ${ }^{1,2}$

\section{${ }^{1}$ Vorozhtsov Novosibirsk Institute of Organic Chemistry, 630090, Russia, Novosibirsk, Lavrentjev ave., 9 \\ ${ }^{2}$ Novosibirsk State University, 630090, Russia, Novosibirsk, Pirogov str., 1 \\ ${ }^{3}$ Institute of Cytology and Genetics, Siberian Branch of Russian Academy of Sciences, 630090 Russia, Novosibirsk, Lavrentjev ave., 10}

Introduction

The search for inhibitors of key enzymes of DNA repair is a promising area of medicina chemistry, which opens up various perspectives for the development of effective methods for the treatment of cardiovascular, neurodegenerative, and oncological diseases. Malignant lioma, which is most common in adults, is an example of cancer. The current standard of care for patients recently diagnosed with a disease such as glioblastoma multiforme (GBM, grade IV glioma) includes maximal surgical resection of the tumor, postoperative chemoradiation therapy with temozolomide, and subsequent adjuvant therapy with temozolomide.

For the treatment of cancer, inhibitors of the reparation systems are needed. One of the specific targets is the enzyme tyrosyl-DNA phosphodiesterase 1 (TDP1). Inhibition of this enzyme can increase the sensitivity of tumor cells to drugs used to treat cancer.

Earlier, the substance KS-389 was obtained, which in in vitro experiments showed a synergistic effect against glioblastoma cells when was administrated together with temozolomide [1].

To find the optimal dosage form of the compound, the method of its administration and dosage of the substance, it is necessary to study its pharmacokinetics.

However, this study has not been completed to date. This work is devoted to the development of a method for the quantitative determination of the agent KS-389 in whole blood of mice for further study of its pharmacokinetic profile.

\section{Materials and methods}

The substance KS-389 was synthesized earlier in the laboratory of physiological substances of the NIOCh SB RAS and purified by column chromatography on silica gel. The compound purity was over $98 \%$ by HPLC-UV.

In our study, we developed a fast, selective and sensitive procedure for quantitation of KS-389 in whole blood of mice using HPLC-MS/MS. The internal standard was a solution of 2,5-bis (4diethylaminophenyl) $-1,3,4-$-oxadiazole $(2,5-\mathrm{BDPO})$ in methanol with a concentration of $20 \mathrm{ng} / \mathrm{ml}$ for processing blood samples.
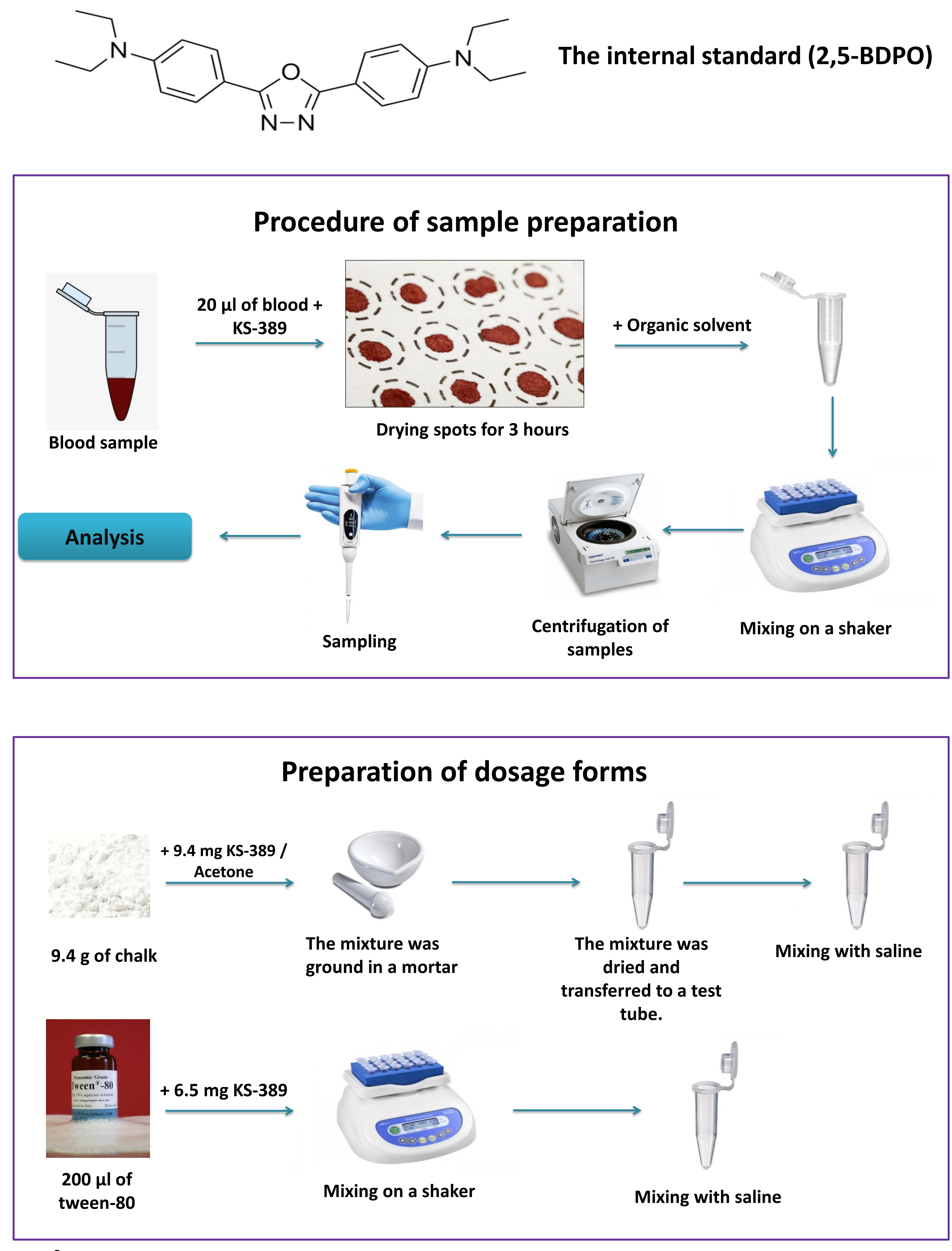

References

1. Kovaleva K. et al. Dehydroabietylamine Ureas and Thioureas as Tyrosyl-DNA Phosphodiesterase 1 Inhibitors That Enhance the Antitumor Effect of Temozolomide on Glioblastoma Cells //Journal of natural products. - 2019. - v. 82. - №. 9. - p. 2443-2450.
Results

To plott the calibration curve, we used whole blood samples containing the agent KS-389 in concentrations of $2,5,10,20,50,100,200,800,2000 \mathrm{ng} / \mathrm{ml}$. This range covers typical values for both low and high concentrations of biologically active drugs administered in doses of several $\mathrm{mg} / \mathrm{kg}$. A linear calibration dependence was obtained in the concentration range mentioned above.

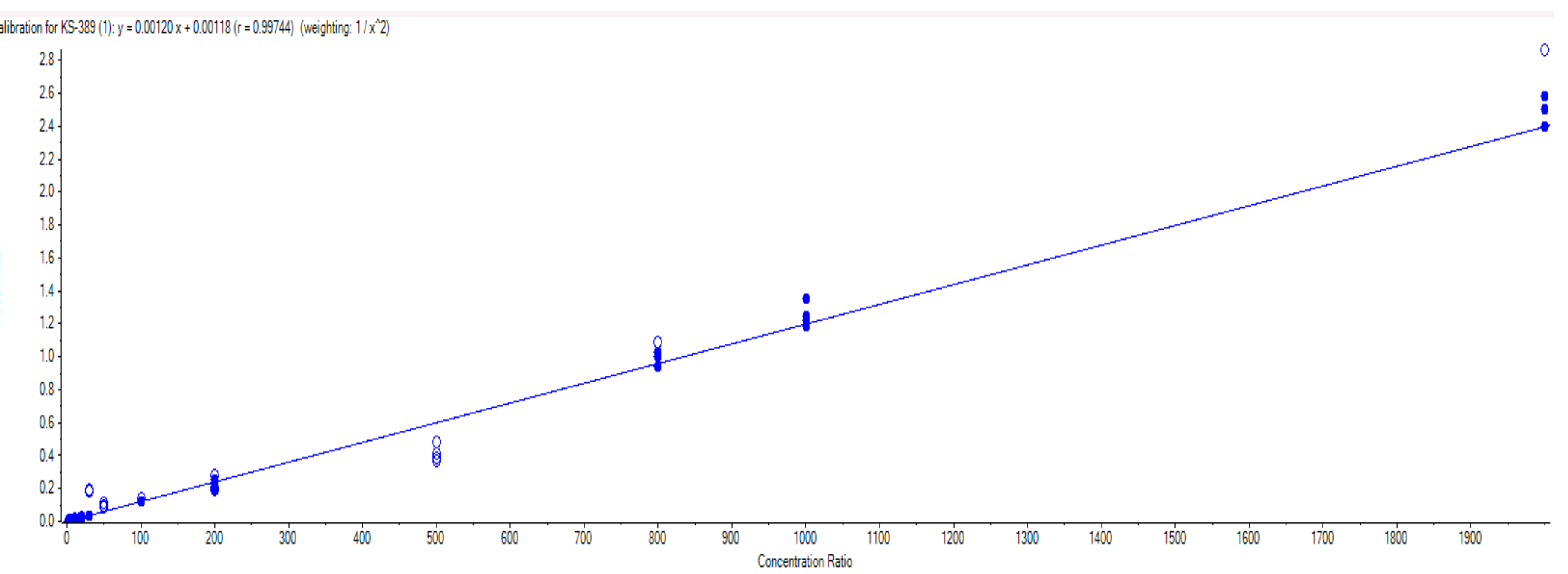

Using the developed method, a preliminary pharmacokinetics study of the substance was carried out.

When the suspension of KS-389+chalk was administered, the maximum concentration of the agent was observed after 30 minutes and corresponded to $23 \mathrm{ng} / \mathrm{ml}$. After that, the concentration of the agent decreased to $2-3 \mathrm{ng} / \mathrm{ml}$. This concentration remained in the blood of the animal for a long time, and the maximum observed on the graph can be caused by several stages of absorption of the substance into the blood of animals. Perhaps, when the suspension is administered to animals, it is incompletely dissolved in the stomach, followed by the release of a certain amount of the substance and its absorption into the blood, after which the chalk carrier moves into the intestine, where additional absorption of the substance into the blood occurs.

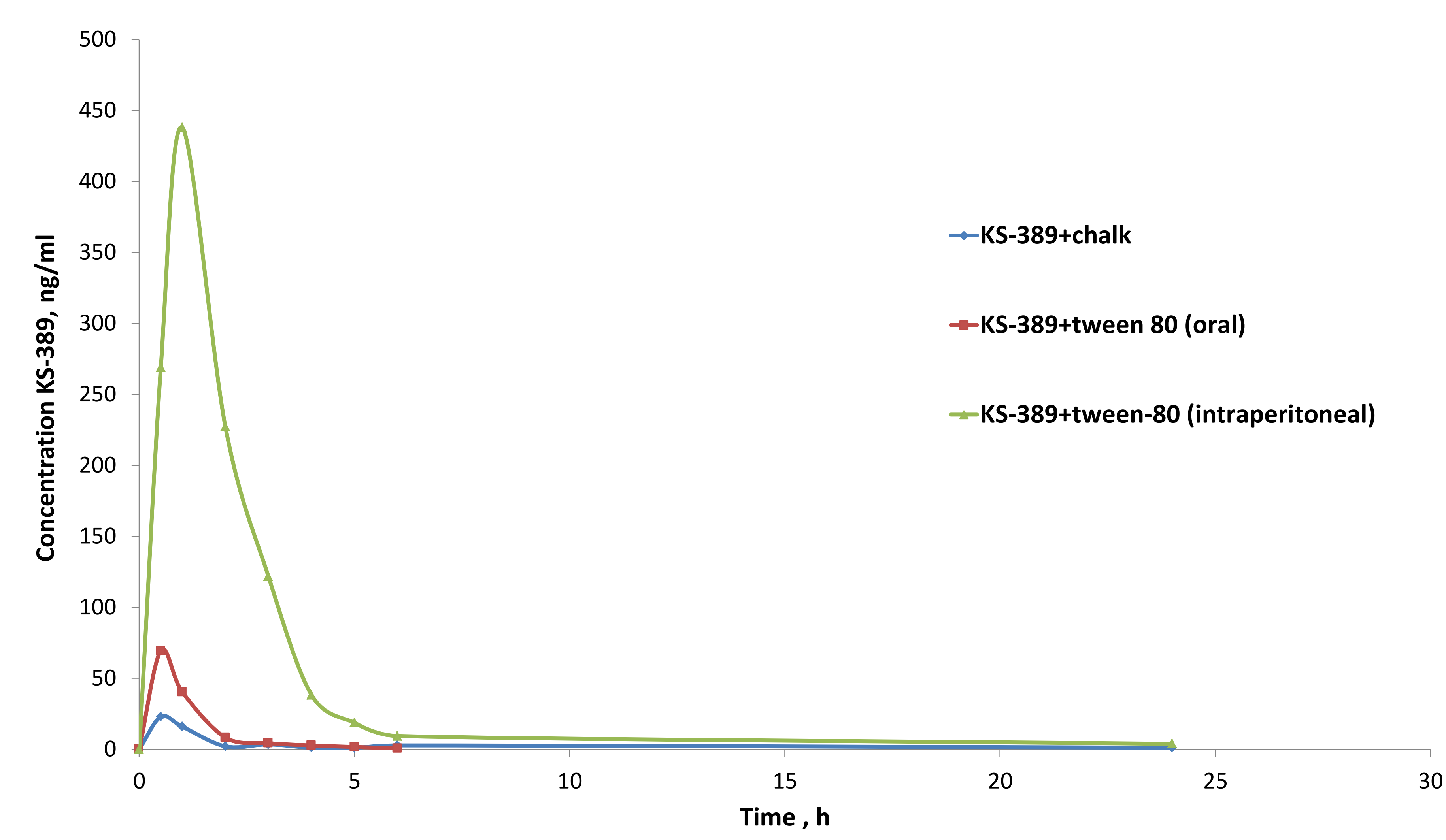

Oral administration of a suspension of the substance with tween-80 provides a slightly bette bioavailability of the substance in the body compared to the use of chalk. The maximum concentration of KS-389 is reached after 30 minutes and corresponds to about $70 \mathrm{ng} / \mathrm{ml}$. After reaching the maximum, there is a gradual decrease in the concentration of the substance in the blood, and the almost complete elimination of the substance from the body occurs after $5.5-6$ hours.

With intraperitoneal administration of a dosage form based on tween-80 to an animal, the maximum concentration of $\mathrm{KS}-389$ is reached $\mathbf{1 5 - 2}$ hours after and is about $450 \mathrm{ng} / \mathrm{ml}$. Over time, the concentration of the substance in the blood gradually decreases and after about 6 hours it is almost completely eliminated from the body.

When the dosage form based on tween-80 was administrated intraperitoneally, the active concentration of the substance in the blood was at the level of several hundred $\mathrm{ng} / \mathrm{ml}$, which can provide its therapeutic effect with sufficient effectiveness. In addition, the data allow the use of a new route of agent administration for detailed pharmacokinetic studies. 